\title{
A Unified Characterization of Reproducing Systems Generated by a Finite Family
}

\author{
By Demetrio Labate
}

\begin{abstract}
This article presents a general result from the study of shift-invariant spaces that characterizes tight frame and dual frame generators for shift-invariant subspaces of $L^{2}\left(\mathbb{R}^{n}\right)$. A number of applications of this general result are then obtained, among which are the characterization of tight frames and dual frames for Gabor and wavelet systems.
\end{abstract}

\section{Introduction}

The aim of this article is to provide an unified approach to the characterization of a large class of systems satisfying a reproducing formula of the form

$$
f=\sum_{i \in \mathcal{I}}\left\langle f, \psi_{i}\right\rangle \psi_{i}
$$

or, more generally,

$$
f=\sum_{i \in \mathcal{I}}\left\langle f, \phi_{i}\right\rangle \psi_{i}
$$

where $f, \psi_{i}, \phi_{i}, i \in \mathcal{I}$, belong to $L^{2}\left(\mathbb{R}^{n}\right)$. The kind of reproducing systems that we will consider are generated by the action of translations, dilations, and modulations on a finite family of functions. To keep the notation to a minimum and focus on the main ideas that we will present in this article, let us restrict our attention, for the moment, to one-dimensional systems generated by a single function. The Gabor system, for example, is generated by the action of the translations $T_{c k}, k \in \mathbb{Z}, c>0$, and modulations $M_{b m}, m \in \mathbb{Z}, b>0$, on a function $\psi \in L^{2}(\mathbb{R})$, where $T_{c k} \psi(x)=\psi(x-c k)$ and $M_{b m} \psi(x)=e^{2 \pi i b m x} \psi(x)$. The system thus obtained is $\left\{\psi_{i}\right\}_{i \in \mathcal{I}}$ $=\left\{M_{b m} T_{c k} \psi\right\}_{m, k \in \mathbb{Z}}$, where the indexing set, in this case, is $\mathcal{I}=\{(m, k): m, k \in \mathbb{Z}\}$. Wavelets are obtained in a similar way, with the dilations $D_{2^{j}}$, where $D_{2^{j}} \psi(x)=2^{j / 2} \psi\left(2^{j} x\right), j \in \mathbb{Z}$, replacing the modulations. The system thus obtained has the form $\left\{\psi_{i}\right\}_{i \in \mathcal{I}}=\left\{D_{2^{j}} T_{c k} \psi\right\}_{j, k \in \mathbb{Z}}$, where $\mathcal{I}=\{(j, k): j, k \in \mathbb{Z}\}$, and is often referred to as an affine system. In the case of the Gabor system, the order of the modulation and translation operators can be reversed; however, this

\footnotetext{
Math Subject Classifications. 42C15.

Key Words and Phrases. affine systems, Gabor systems, shift-invariant spaces, wavelets.
} 